\title{
HACIA LA EVIDENCIA BASADA EN LA PRÁCTICA EN PSICOTERAPIA
}

\section{TOWARDS PRACTICE-BASED EVIDENCE IN PSYCHOTHERAPY}

\author{
Alberto Gimeno Peón \\ Psicólogo Clínico, práctiva privada. Gijón, España \\ ORCID: https://orcid.org/0000-0002-6559-5821
}

\author{
César Mateu Hernández \\ Psicólogo Clínico, Doctor en Psicología. Unidad de Salud Mental Burjassot. \\ Departamento Arnau de Vilanova-Lliria, Valencia. España \\ Facultad de Psicología, Universidad Católica de Valencia. España \\ ORCID: https://orcid.org/0000-0003-3063-2837
}

Cómo referenciar este artículo/How to reference this article:

Gimeno-Peón, A. y Mateu, C. (2020). Hacia la Evidencia Basada en la Práctica en Psicoterapia. Revista de Psicoterapia, 31(117), 179-194. https://doi.org/10.33898/rdp.v31i117.387

\section{Resumen}

La práctica basada en la evidencia en psicología ha permitido mostrar la eficacia de multitud de procedimientos y tratamientos psicoterapéuticos dirigidos al abordaje de toda una serie de problemas psicológicos y de los denominados trastornos mentales. Sin embargo, siguen existiendo algunas preguntas abiertas respecto a cuáles son los factores que más influyen en los resultados de la terapia, junto con la dificultad de aplicar el conocimiento adquirido en condiciones controladas a la práctica clínica a la que se enfrentan los especialistas en contextos naturales. En este trabajo se revisan estas cuestiones, así como algunos problemas metodológicos y teóricos relacionados con la práctica basada en la evidencia. Además, se plantea el concepto de la evidencia basada en la práctica como una forma de tender puentes entre las situaciones de investigación más controladas y la práctica rutinaria, revisando y resaltando aquellos elementos del proceso terapéutico que contribuyen a mejorar los resultados de la psicoterapia.

Palabras clave: práctica basada en la evidencia, evidencia basada en la práctica, psicoterapia, psicología clínica, investigación orientada por la práctica.

\begin{abstract}
Evidence-based practice in psychology has shown the effectiveness of a multitude of psychotherapeutic procedures and treatments aimed at addressing a whole series of psychological problems and the so-called mental disorders. However, there are still some open questions regarding which factors influence the results of therapy the most, along with the difficulty of applying the knowledge acquired under controlled conditions to the clinical practice faced by specialists in natural contexts. This paper reviews these questions, as well as some methodological and theoretical problems related to evidence-based practice. In addition, the concept of practice-based evidence is proposed as a way to build bridges between the most controlled research situations and routine practice, reviewing and highlighting those elements of the therapeutic process that contribute to improving the results of psychotherapy.

Keywords: evidence-based practice, practicebased evidence, psychotherapy, clinical psychology, practice oriented research.
\end{abstract}

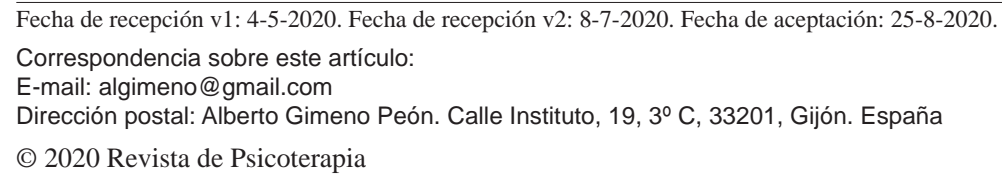


El desarrollo de la investigación acerca de la psicoterapia, así como de su aplicación en el ámbito clínico ha sido innegable en las últimas décadas. Esta ha dejado más que demostrada su eficacia a la hora de abordar un gran número de problemas humanos, habitualmente categorizados como "trastornos mentales" (Lambert, 2013). Gran parte de este mérito lo tiene el haber sido capaz de adoptar los criterios de la medicina basada en la evidencia, dando lugar a la práctica basada en la evidencia en psicología (PBEP, en adelante; American Psychological Association, 2006), lo que ha permitido mostrar el valor de los tratamientos psicológicos, especialmente en cuanto al bienestar de la población se refiere.

Sin embargo, a pesar de sus bondades, el modelo actual de PBEP adolece de algunos puntos débiles que merecen ser objeto de reflexión. Entre ellos, destaca la enorme dificultad a la hora de trasladar toda la tecnología y conocimiento adquirido mediante estudios controlados al contexto clínico real, la práctica rutinaria de los especialistas. Algunos autores han criticado los postulados de la PBEP por considerarlos reduccionistas (Berg, 2019; Berg y Slaattelid, 2017), por ejemplo. Otros se han referido a la brecha existente entre investigación y práctica clínica (Castonguay et al., 2019; Fernández-Álvarez et al., 2020) y a la falta de consenso con respecto a los principios que operan en psicoterapia, en parte agravado por la tendencia a privilegiar nuevos términos o conceptos que tratan de reinventar viejas ideas (Goldfried, 2019). Estas y otras circunstancias han llevado a la propuesta de un enfoque conocido como evidencia basada en la práctica, cuyo objetivo no es sustituir el paradigma de la PBEP ni contraponerse al mismo, sino complementarlo y enriquecerlo, acercándolo a las situaciones diarias que los psicólogos clínicos deben afrontar en su propio ámbito de trabajo.

El objetivo de este trabajo es revisar el concepto de PBEP, incidiendo en algunas críticas y limitaciones con respecto a su aplicación a la psicoterapia, así como señalando aquellos factores que han demostrado ser más importantes de cara a un desempeño más efectivo. Finalmente, se presentará el concepto de evidencia basada en la práctica, citando algunos ejemplos reales.

\section{La práctica basada en la evidencia en psicología}

Décadas de estudios en psicoterapia nos han permitido llegar a una serie de conclusiones, recogidas en el trabajo fundamental de Lambert (2013). La básica, y de mayor interés, es la de su más que demostrada eficacia, presentando en términos generales un tamaño del efecto alto, de alrededor de 0.80 . Y eso es así en el caso de multitud de problemas psicológicos, logrando cambios estables que suelen mantenerse a largo plazo, incluso cuando se trata con lo que generalmente se categoriza como "trastornos mentales graves".

Así mismo, diversas investigaciones han hallado resultados más problemáticos y, en algunos casos, incluso polémicos. Es especialmente intenso el debate acerca de la ausencia de diferencias significativas, en términos generales, con respecto a la eficacia de diversos modelos y tipos de tratamiento psicológico (Wampold e 
Imel, 2015), aquello que se ha venido denominando como "el veredicto del Pájaro Dodo", y que todavía hoy genera debates acalorados entre investigadores y clínicos (González-Blanch y Carral-Fernández, 2017; Luborsky et al., 2002). Si bien es cierto que si se habla en términos propios de un modelo médico (utilizando diagnósticos basados en la supuesta presencia de trastornos) algunos tipos de tratamiento han mostrado ser más eficaces que otros, se podrían cuestionar varios factores al respecto, desde la fiabilidad de los criterios diagnósticos hasta el tipo de intervenciones utilizadas para demostrar la pretendida superioridad de un tratamiento determinado. Por otro lado, algunas revisiones cuestionan la ausencia de diferencias significativas entre enfoques de terapia, así como el valor dado a los factores comunes a estos (Cuijpers et al., 2019).

Lo que resulta incuestionable es que, a pesar de haber avanzado tanto en la investigación en psicoterapia y haber engrosado drásticamente los listados de tratamientos con apoyo empírico, la variabilidad de resultados sigue siendo enorme. Que el margen de mejoría es amplio lo indican datos como el porcentaje de personas que no muestran progreso alguno durante la terapia (entre un 30-50\%; Lambert, 2013), una tasa media de abandonos que oscila en torno al 20\% (Swift y Greenberg, 2012) o el hecho de que entre el $5 \%$ y el $10 \%$ de las personas adultas y entre el $14 \%$ y el $24 \%$ de niños empeoran de forma significativa durante la intervención (Lambert, 2013). Por supuesto, lo que llamamos "abandono prematuro" no siempre es señal de un resultado negativo; en muchas ocasiones, la persona considera que ya ha obtenido la ayuda suficiente que esperaba y no ve necesario acudir a más sesiones (Roe et al., 2006; Westmacott y Hunsley, 2010). Así mismo, que alguien empeore mientras acude a terapia no implica que sea el propio tratamiento el responsable de esa progresión negativa; simplemente, puede decirse que ya había una trayectoria de deterioro previa a comenzar la psicoterapia y que ésta ha sido incapaz de detener y mucho menos revertir. Por desgracia, la disciplina todavía adolece de algunas dificultades a la hora de recoger resultados de una forma del todo fiable (Langkaas et al., 2018). De todas maneras, no conviene caer en interpretaciones autocomplacientes sobre estos resultados aparentemente adversos y descartar la autocrítica, la cual es necesaria para poder estudiar estos fenómenos y encontrar métodos prácticos que permitan aumentar la eficacia general de los clínicos. En relación con todo lo anterior, es también importante resaltar que los porcentajes citados (en cuanto a casos que mejoran, abandonan o empeoran) varían mucho en función de un factor clave: el profesional encargado de llevar a cabo la psicoterapia. Existen diferencias significativas en los resultados de la intervención en función de quien sea el especialista responsable (Barkham et al., 2017), como se verá más adelante.

Aunque el concepto de PBEP parece muy arraigado entre clínicos, investigadores y estudiantes de psicología, cabe preguntarse hasta qué punto realmente se comprende su sentido en realidad, así como todo lo que abarca. Pareciera que toda la PBEP se redujera a elegir un tratamiento específico de un listado y aplicarlo cuando detectemos un trastorno concreto. Sin embargo, la idea que subyace a la 
práctica basada en la evidencia (no solo en psicología) es mucho más amplia y compleja. La PBEP se define como "la integración de la mejor evidencia disponible con la pericia clínica, en el contexto de las características, cultura y preferencias del paciente" (American Psychological Association, 2006). Estos tres factores se han representado, con fines didácticos, como las tres patas de un taburete, aunque realmente no son elementos independientes que operan al margen los unos de los otros, sino que son variables inseparables que se influyen mutuamente y muestran cierto solapamiento, tal y como las representan, por ejemplo, Mulder et al. (2017), y donde vemos que juegan un papel importante otros factores como la toma de decisiones colaborativa, la formulación personalizada de cada caso o la monitorización de resultados, entre otros. Aquí tienen cabida también la investigación de resultados, la investigación de procesos y la investigación básica sobre aquellos aspectos que influyen en el bienestar personal.

Como se indicaba en la introducción, que la psicología aceptara las reglas del juego de la medicina basada en la evidencia ha supuesto un hito importante que ha permitido dotar de una base científica a las actividades del profesional dedicado a la psicoterapia. Fruto de ello, han aparecido un elevado número de tratamientos psicológicos que han logrado alcanzar el estatus de "tratamientos con apoyo empírico", siendo estos de muy variada procedencia teórica y aplicables a toda una miríada de problemas humanos (incluso en aquellos considerados más graves, como los trastornos psicóticos). Se podría decir que, dentro de las actividades sanitarias, han colocado a la psicoterapia (y con ello, la labor de los psicólogos clínicos) en el lugar que se merece, respaldados por una metodología suficientemente sólida. Sin embargo, esta misma metodología, adolece de una serie de limitaciones y problemas, de entre los que se podrían destacar (aunque no son los únicos) los siguientes: - $\quad$ Crisis de replicabilidad en psicología clínica: Tackett et al. (2019) han señalado una serie de motivos de preocupación con respecto a los estudios en psicología clínica, alarmados ante la crisis de replicabilidad, que no solo afecta al ámbito de la terapia, sino a otras aplicaciones de la psicología. Los problemas principales parecen estar relacionados con las limitaciones inherentes al uso de la significación estadística como criterio de eficacia, pero también incluyen cuestiones tales como la falta de fiabilidad de los diagnósticos que siguen un modelo médico, los sesgos de publicación (con una sobrerrepresentación de estudios con resultados positivos, hasta el 96\% de los publicados), prácticas de investigación cuestionables o falta de validez externa de las investigaciones controladas y la dificultad asociada de trasladar los resultados al contexto clínico real.

- $\quad$ Crisis en los tratamientos psicológicos con apoyo empírico: los criterios para considerar que un tratamiento goza de suficiente apoyo empírico son cuestionables. Wampold e Imel (2015) han señalado cómo en numerosas ocasiones se han comparado sus efectos con una supuesta terapia que, realmente, no se había diseñado como lo que estos autores 
denominan una psicoterapia bona fide. Más aún, una revisión meta-científica reciente ha puesto de manifiesto problemas importantes en gran parte de los estudios sobre la eficacia de los tratamientos con apoyo empírico (Sakaluk et al., 2019). Estos autores hallaron que, cuando se analizan los resultados de una manera más rigurosa, la evidencia otorgada a muchas de estas terapias es menor de lo que se considera en listados como el de la Asociación Americana de Psicología (American Psychological Association, 2016). Casi el 50\% de los estudios analizados contenían al menos un error en cuanto al análisis estadístico y su interpretación; de hecho, en el $13 \%$ de las publicaciones se detectaron errores graves que socavaban las conclusiones a las que llegaban los autores.

- $\quad$ Problemas epistemológicos y pragmáticos al adoptar el modelo médico en psicología: a la hora de elaborar guías de tratamientos psicológicos eficaces se han seguido las mismas directrices que las empleadas en las guías de intervenciones médicas, asumiendo que se rigen por los mismos principios y abordan elementos similares. Pero, como señalan PérezÁlvarez et al. (2003), las reglas que rigen la aplicación de los tratamientos psicológicos deben ser diferentes a las de los tratamientos médicos, ya que se diseñan para tratar problemas psicológicos, no biológicos ni neurológicos; protocolizar una terapia no soluciona completamente el problema del control experimental; y no es suficiente con valorar la reducción de síntomas como criterio de éxito, ya que hay otro tipo de cuestiones relacionadas con el bienestar y lo que se considera mejoría en el campo de la salud mental que son igualmente (o más) importantes. Existen numerosas investigaciones que demuestran que las psicoterapias pueden mejorar la calidad de vida en la mayoría de los trastornos mentales. Sin embargo, hay relativamente poca investigación disponible sobre resultados definidos por el propio paciente. De hecho, los pacientes generalmente no solo acuden a la terapia para obtener alivio de los síntomas, sino también para abordar otros problemas personales que repercutan de manera positiva aumentando su calidad de vida (Cuijpers, 2019). Algunos autores, dado que el paciente es el principal agente de cambio, proponen un modelo conceptual de "buen resultado" en psicoterapia. Desde la perspectiva de los pacientes, un buen resultado puede entenderse como sentirse empoderado, encontrar el equilibrio personal y poder continuar afrontando los problemas, lo que indica que se trata de un proceso continuo y variable según la experiencia personal (De Smet et al., 2020).

\section{¿La mejor evidencia disponible?}

Siendo este factor, probablemente, el que más se identifica con la PBEP, llama la atención la falta de guías que especifiquen claramente cuál es la mejor evidencia disponible, más allá de un listado de tratamientos dirigidos a abordar trastornos 
mentales con nombre propio. Al mismo tiempo que el listado crece, lo hace también el número de publicaciones sobre psicoterapia y la facilidad de acceso a las mismas. Dentro de la confusión que puede suponer para muchos clínicos (especialmente los más inexpertos) encontrarse con estudios que afirman demostrar unos resultados $\mathrm{y}$, al poco tiempo, otro estudio que lo cuestiona, no sería de extrañar que la manera que se tenga de responder a esta cuestión sea mediante un sesgo de confirmación, que induce al profesional a confiar más en aquellos trabajos que confirman sus teorías, modelos y visión de la psicoterapia, por encima de la validez empírica de los mismos.

La propia APA (2006) indica que, mientras que un enfoque que sólo considera los tratamientos con apoyo empírico trata de responder a la pregunta de si un tratamiento concreto funciona bajo unas circunstancias específicas (y se centra, por tanto, en la terapia), en la PBEP se comienza por el paciente con el objetivo de dilucidar qué pruebas son aquellas que ayudarán al clínico a lograr el mejor resultado para esa persona, en su contexto real. Para este fin, no solo son útiles los ensayos clínicos aleatorizados y los meta-análisis, sino que también cabe considerar otro tipo de diseños de investigación, que también se incluyen dentro de las prácticas basadas en la evidencia como los estudios de caso y la investigación cualitativa, entre otros, ya que son fuentes de información que no deben ser descartadas por no tener el nivel de control experimental de los ensayos clínicos (Heinonen y Nissen-Lie, 2020).

Conocer la mejor evidencia disponible no se limita a estar al tanto de los tratamientos con apoyo empírico, sino que implica saber qué factores de la terapia tienen una influencia en los resultados y de qué manera lo hacen. Norcross y Lambert (2019) han puesto de manifiesto que el método de tratamiento supone solo un $10 \%$ de la varianza de los resultados en psicoterapia y que otros elementos tienen un papel relevante: características y recursos del paciente(30\%), la alianza terapéutica (15\%), características del clínico (7\%), otros factores (3\%); quedando todavía un 35\% de varianza no explicada. Por supuesto, esta separación es igual de artificial que la de los elementos de la PBEP. Técnicas, método, alianza, características del profesional y del paciente no son elementos que puedan existir de forma independiente, sino que interaccionan entre sí dinámicamente, estableciendo relaciones complejas cuyos efectos no pueden separarse de forma pura. La cuestión a resaltar aquí es que hay toda una serie de factores que el psicólogo clínico debe conocer, los cuales son apoyados por la evidencia en mayor medida que la mayoría de los tratamientos con apoyo empírico. Se trata de elementos tales como la alianza terapéutica, la obtención y uso de feedback, el manejo de la contratransferencia, la reparación de rupturas en la alianza y diferentes métodos de adaptación de la terapia a ciertas características de los pacientes, entre muchas otras, cuya evidencia recogen Norcross y Wampold (2018). En realidad, estos factores representan un 14\% de la varianza del resultado en psicoterapia, ya que el $86 \%$ restante se atribuye a los factores propios de la vida del cliente que no tienen ninguna relación con lo que ocurre en la psicoterapia. Aunque a simple vista un $14 \%$ pueda parecer un porcentaje pequeño, su papel es 
de crucial importancia, dado que todos los factores que influyen en el proceso del tratamiento psicológico se engloban en ese pequeño, y a la vez tan enorme, $14 \%$ (Norcross y Lambert, 2019). En la tabla 1 se muestra el peso de cada factor dentro del porcentaje anteriormente mencionado (Duncan, 2014).

Tabla 1. Porcentaje de Varianza Explicada por Factores que Influyen en la Psicoterapia

\begin{tabular}{lc}
\hline FACTORES & $\begin{array}{c}\text { \% DE VARIANZA DE } \\
\text { RESULTADO EXPLICADA }\end{array}$ \\
\hline Efectos del terapeuta & $36 \%-57 \%$ \\
\hline Alianza & $36 \%-50 \%$ \\
\hline Feedback & $21 \%-42 \%$ \\
\hline $\begin{array}{l}\text { Modelo/Técnica: Efectos generales (racional y } \\
\text { ritual), Expectativa del cliente (esperanza, placebo), } \\
\begin{array}{l}\text { Confianza en el terapeuta. } \\
\text { Modelo/Técnica: Efectos específicos (diferencias } \\
\text { entre modelos) }\end{array}\end{array}$ \\
\hline
\end{tabular}

\section{El profesional eficaz y su formación}

Aunque el interés acerca de las características que convierten a un clínico en un profesional eficaz ha estado presente en la literatura desde hace décadas, en los últimos años el desarrollo de la metodología ha permitido estudiar el fenómeno de los efectos del terapeuta (el porcentaje de la varianza de los resultados que depende del profesional y no de otras variables de la psicoterapia) en mayor detalle y de forma más fiable. Como es habitual, existe cierta variabilidad entre estudios, pero se puede hablar de que esta variable influye en entre un 5\% y $8 \%$ de los resultados del tratamiento (Barkham et al., 2017). Este porcentaje, además, es mayor cuando se trata de casos más graves. Este fenómeno indica, en líneas generales, que: hay profesionales que son mejores que otros; esta diferencia es un hecho estable en el tiempo y frente a diferentes tipos de casos; los clínicos más efectivos logran mayores porcentajes de mejoría, reduciendo las tasas de abandonos prematuros y de casos que empeoran de forma significativa; y para todo esto emplean un número menor de sesiones, lo que los hace más eficientes (Castonguay y Hill, 2017).

No es de extrañar que la pericia sea un aspecto a considerar en psicoterapia. $\mathrm{Al}$ fin y al cabo, la persona del profesional es una herramienta imprescindible mediante la que se pone en marcha el tratamiento, que interactúa con los pacientes, que influye y, a la vez, es influido por ellos, por lo que es importante que esté "en forma" si quiere dar un buen servicio. Se ha tratado de dilucidar qué características tienen los clínicos que muestran un desempeño superior y no se ha encontrado que la diferencia esté relacionada con variables tales como la experiencia, la edad, la orientación teórica, la psicoterapia personal o la supervisión, entre otros aspectos, tal y como muestran varias revisiones (e.g.: Chow, 2014; Castonguay y Hill, 2017; Prado-Abril et al., 2019; Wampold et al., 2017). Wampold et al. (2017) concluyen que hay evidencia para considerar que los clínicos más eficaces reúnen, al menos, 
cuatro características: son capaces de establecer buenas alianzas terapéuticas con un amplio rango de personas de diferentes características (y niveles de gravedad); muestran lo que algunos autores han denominado habilidades facilitadoras del cambio (Anderson et al., 2009) y que tienen que ver con factores como la empatía, calidez o fluidez verbal, entre otros; dudan acerca de su propio desempeño, pero con un sentido del self positivo (Nissen-Lie et al., 2017); y, por último, comienza a proponerse que el tipo de práctica que el psicólogo utiliza para mejorar, como parte de su desarrollo profesional, así como el tiempo dedicado a la misma, puede estar también asociado a los efectos del terapeuta.

Habida cuenta de la importancia no solo de lo que hace el profesional de la psicoterapia, sino también del cómo lo hace, parece sensato estudiar si la formación que estamos ofreciendo a los clínicos es la más adecuada. Más allá de los contenidos formativos, toca centrarse en los métodos de enseñanza y aprendizaje de habilidades terapéuticas comunes a diferentes formas de intervención, así como de destrezas más específicas centradas en técnicas y modelos concretos. Una vez que hemos puesto a prueba diferentes tratamientos, llega el momento de hacer lo propio con la formación para comprobar si es eficaz a la hora de alcanzar el objetivo: mejorar el desempeño de los clínicos a la hora de hacer psicoterapia. En ese sentido, están surgiendo interesantes propuestas. Una de ella es la denominada práctica deliberada (Erickson et al., 1993), un tipo de entrenamiento que se ha estudiado previamente en otras disciplinas y que, algunos autores están tratando de adaptar a la formación en psicoterapia (Prado-Abril et al., 2019; Rousmaniere, 2016, 2019). En términos generales, implica un método en el que un supervisor observa una grabación de trabajo real del clínico para poder darle feedback preciso y señalar aspectos a mejorar, acordando una serie de prácticas que permitan al supervisado mejorar su desempeño e ir, progresivamente, refinando las habilidades diana. Otros autores se han referido a la práctica reflexiva (Stedmon y Dallos, 2009) o a la práctica personal (Bennet-Levy, 2019), siendo especialmente sugerente esta última propuesta. Bennet-Levy (2019) ofrece un modelo parsimonioso en el que se tienen en cuenta tanto los aspectos más técnicos de la formación del profesional como aquellas características y circunstancias personales que influyen en el buen quehacer diario del clínico, tratando de fomentar la reflexión y la conciencia de la identidad profesional y la identidad personal e integrándolas por medio de una serie de acciones, que permitan actuar a modo de puente entre los métodos más tradicionales de formación y los componentes de la práctica personal en sí mismos.

Todo lo anterior parece indicar la importancia de la formación en habilidades terapéuticas, no solo las básicas, sino también algunas más avanzadas, así como las específicas del propio método de intervención y las comunes a otras prácticas eficaces. No sería descabellado pensar que en poco tiempo se hable de "métodos de formación en psicoterapia basados en la evidencia”. Un paso previo deberá consistir, necesariamente, en revisar el tipo de aprendizaje que están siguiente los futuros profesionales que se dediquen en la psicoterapia. Un trabajo que ya se ha 
comenzado a hacer en el caso de la psicología clínica y de su formación sanitaria especializada (Montejano y García, 2019; Prado-Abril et al., 2017; Prado-Abril et al, 2019).

\section{La persona que acude a terapia: motor del cambio}

Todo lo señalado hasta ahora no debe hacer perder de vista al clínico algo fundamental: el protagonista del cambio, y por tanto de la terapia, sigue siendo la persona que acude a consulta. Ningún tratamiento, por mucho apoyo empírico con el que cuente, ni ningún profesional, por bueno que sea, son efectivos si no tienen en consideración el tercer factor de la PBEP: la persona, con sus características psicológicas, sus valores, sus preferencias y el contexto en el que tiene lugar su historia. Tal y como señalan Bohart y Wade (2013):

es el cliente, más que el terapeuta, quien pone en marcha el proceso de cambio. Si el cliente no absorbe, utiliza y sigue adelante con los esfuerzos del terapeuta, no sucede nada. ¡Más que discutir acerca de si la terapia funciona o no, deberíamos plantearnos si el cliente funciona! En este sentido, necesitamos un cambio en nuestra forma de pensar sobre la eficacia de la psicoterapia. Los clientes no son objetos inertes a los que se les administran técnicas. No son variables dependientes sobre las que operan variables independientes. (p. 219)

Este es otro de los problemas con los que se encuentra la psicoterapia cuando trata de mimetizar el modelo médico: se pierde de vista que la persona no es un receptor pasivo de nuestra pericia y tratamientos basados en la evidencia, cuyo único papel es seguir a rajatabla las indicaciones del psicólogo clínico, ante las que se espera que reaccione como si fuera una molécula; ésto no es muy diferente a tratar una depresión utilizando fármacos que reequilibren los niveles de serotonina, por ejemplo. Una intervención enfocada de esta manera patologiza en exceso los problemas humanos y deja al paciente indefenso, lo desautoriza y minusvalora sus propios recursos; le quita el protagonismo de su propia vida.

A pesar de la tendencia, en algunos ámbitos, a centrarse cada vez más en variables "neuro" (Slife et al., 2010; parece como si recurrir a esa palabra diera una capa extra de apariencia científica a la psicoterapia), lo cierto es que hay evidencias que apuntan a multitud de variables psicológicas asociadas a las personas que acuden a terapia y que tienen un peso significativo en los resultados. La revisión de Bohart y Wade (2013) recoge las más importantes, tratándose de aspectos que no residen en el cerebro, sino en la psicología, en la interacción con otras personas y el mundo.

Por este motivo, se han llevado a cabo numerosos estudios cuya finalidad es encontrar las maneras más eficaces de adaptar la psicoterapia a ciertas características de las personas, aquellas sobre las que existe evidencia de su contribución a los resultados. Norcross y Wampold (2019) han revisado la importancia de diferentes métodos de adaptación, entre los que se incluyen la cultura de la persona (Soto et al., 2018), sus preferencias (Swift et al., 2018), nivel de reactancia (Beutler et al., 
2018a), estilo de afrontamiento (Beutler et al., 2018b), estilo de apego (Levy et al., 2018) o motivación para el cambio (Krebs et al., 2018).

\section{Modelos, teorías y prejuicios}

A pesar de que muchas voces, apoyadas en la investigación, insisten en señalar que la orientación teórica o modelo de terapia no es tan relevante como otros aspectos de la interacción consultante-psicólogo clínico, todavía muchos profesionales se resisten a esta realidad. Cecchin (2010) ha utilizado el término "prejuicios sistémicos" en referencia al trabajo desde su propio modelo para resaltar que nuestras teorías no dejan de ser hipótesis sobre el funcionamiento humano y, en concreto, sobre lo que es o no patológico y lo que se debe hacer para ayudar a las personas. Pero las teorías no son en sí mismas la realidad. Parece apropiado adoptar este concepto de prejuicios por parte de clínicos de cualquier orientación, no para desvalorizar los modelos conocidos ni dejar de contar con ellos, sino para evitar que un uso inflexible de los mismos lleve a una práctica rígida que termine realizando su propia versión de la historia del lecho de Procusto (utilizar el mismo método de tratamiento y de relación terapéutica con cualquier persona, sin tener en cuenta sus particularidades), lo cual no deja de ser un sesgo del que el profesional debe desear prescindir a toda costa. Una actitud como esta permite dar cabida a la curiosidad como antídoto ante el dogmatismo, abriendo posibilidades para aquellas personas con las que el método favorito del profesional no está dando resultado; previniéndonos así, de recurrir a manidos sesgos confirmatorios que descargan la responsabilidad del fracaso terapéutico sobre los hombros del consultante.

Es complicado, sin embargo, ver desde esta óptica los modelos teóricos cuando se pretende considerar la psicoterapia como la aplicación de una ciencia natural. Resulta difícil no concebir la psicología clínica como una ciencia social, habida cuenta de que se trata de un campo que estudia cómo las personas se relacionan entre sí, con el mundo, y con ellas mismas; donde el contexto, en un sentido amplio, tiene un papel fundamental a la hora de investigar la conducta humana y los fenómenos asociados. Así lo señala Pérez-Álvarez (2019), quien subraya el hecho de que aquello en lo que se centra la psicoterapia no son entidades fijas y naturales, sino interactivas y que dependen, en gran parte, de nuestra propia visión (socialmente construida) del mundo. La psicología es una ciencia social y eso no la hace menos científica ni le quita valor alguno.

La identificación extremadamente rígida con un modelo y su presunción de ser el único verdadero y "científico" está estrechamente relacionada con la intolerancia beligerante hacia otras propuestas y teorías, tachadas en muchas ocasiones de pseudoterapias. Lo cual hace un flaco favor a las personas que solicitan ayuda psicoterapéutica, más aún si se señala de nuevo que hay varios enfoques que han mostrado buenos resultados con respecto a diversos problemas psicológicos. Quizás un camino más productivo implique trabajar en meta-modelos que permitan integrar los aportes de cada una de las tradiciones teóricas que han mostrado eficacia, como 
puede ser el modelo contextual de Wampold (Wampold e Imel, 2015).

No es de extrañar que expertos internacionales en la materia insistan cada vez más en darle a la psicoterapia un aire integrador, lo cual no implica caer en un eclecticismo intuitivo y falto de coherencia, algo que se suele achacar a quienes defienden una actitud integradora de los tratamientos psicológicos sino más bien todo lo contrario, pues aunque hasta la fecha se considera que la división en diferentes escuelas, la falta de especificidad, la duración excesiva del tratamiento, la accesibilidad limitada, el excesivo individualismo ideológico, la brecha entre investigación y práctica clínica, y la tendencia a redescubrir lo que ya se ha descubierto en el pasado han sido los principales factores que han dificultado la integración, algunos autores consideran que, sin estar exenta de problemas y resistencia al cambio, dicha integración puede acabar ocurriendo a largo plazo de manera natural (Goldfried, 2019; Paris, 2013). Más aún teniendo en cuenta que cada vez más psicólogos clínicos se consideran integradores. Por ejemplo, en una muestra compuesta por 350 especialistas y residentes de psicología clínica en España, Prado-Abril et al. (2019) encontraron que el $67.1 \%$ decía trabajar desde un enfoque integrador. Se trata de una muestra en la que la experiencia media es cercana a los 10 años, por lo que no se puede achacar este porcentaje a la falta de práctica clínica real. Es posible que durante el desarrollo profesional de los psicólogos clínicos se vaya haciendo más evidente que trabajar de forma limitada con un único modelo que permanece ajeno a las aportaciones de otros enfoques, no es el mejor planteamiento para ayudar a la población.

Integrar, poner en cuestión los modelos teóricos o relativizar la influencia de la técnica específica no significa decir que es válido cualquier tipo de abordaje. En psicología clínica y en psicoterapia sirven muchas cosas, pero no todo vale. Flexibilidad no implica falta de rigor.

\section{Evidencia basada en la práctica}

La PBEP ha aportado un conocimiento muy importante al campo de la psicoterapia. Sin embargo, gran parte del mismo procede de estudios muy controlados en los que la falta de extrapolación a la práctica clínica rutinaria se ha convertido en un problema. El mayor porcentaje de tratamientos psicológicos se llevan a cabo en un contexto real, donde no es posible una selección y control de variables tan rigurosa como la que muestran los ensayos clínicos. Tampoco se dan, muchas veces, las condiciones idóneas; por ejemplo, en los servicios de salud mental públicos la escasez de personal y otros motivos organizacionales dificulta que la terapia se produzca con la frecuencia recomendada.

La idea de la evidencia basada en la práctica (Margison et al., 2000) está muy ligada al concepto de efectividad, a la práctica clínica rutinaria, con la intención de analizar qué es lo que hacen los profesionales en su trabajo diario y, aún más importante, cuál es el resultado de su intervención. En ese sentido, se considera necesario utilizar medidas de monitorización de resultados de forma sistemática 
que permitan recoger información de calidad. El uso de instrumentos de este tipo ha mostrado mejorar los resultados de la terapia en varios aspectos, especialmente en aquellos casos en los que existe un riesgo de fracaso (Gimeno-Peón et al., 2018b). Dicho de otra forma, la evidencia basada en la práctica implica conocer qué se hace de forma habitual en las consultas de psicoterapia y qué resultado se está obteniendo. Interesa, por lo tanto, recoger información variada, no solo de resultados, sino también sobre procesos, feedback cualitativo de los consultantes, feedback proporcionado por expertos, etc. En cierto sentido se trata de responder a la pregunta "¿cuál es la mejor evidencia disponible en este contexto, con esta población y con este equipo profesional?". Hay dos componentes, en definitiva, a considerar: la efectividad, extrapolación de los resultados a otros servicios de salud mental, dispositivos o contextos, y la práctica clínica en la que se analicen los resultados y se pueden detectar diferencias individuales, subgrupos de consultantes o diferencias entre clínicos (Barkham y Mellor-Clark, 2003).

Al contrario de lo que sucede en los ensayos clínicos, donde se sigue un tratamiento manualizado y muy estructurado, una práctica clínica que se guíe por los resultados, momento a momento, es más reactiva y puede responder de forma inmediata cuando se detectan problemas; esto hace que se abra la posibilidad a buscar alternativas terapéuticas, abriendo un proceso de toma de decisiones colaborativo que termine en un cambio en la intervención. Además, favorece en los equipos una actitud reflexiva, no enjuiciadora, que da incluso la oportunidad de mejorar el desempeño de los terapeutas (Barkham y Mellor-Clark, 2003).

Margison et al. (2000) han señalado que es posible y deseable tender puentes entre la PBEP y la evidencia basada en la práctica, para lo cual proponen algunas guías: identificar qué intervenciones se están llevando a cabo y los motivos para ello; desarrollar la habilidad para usar diferentes tipos de formulación de casos; identificar problemas en la alianza terapéutica, así como formas de solucionarlos; auto-evaluación y supervisión por pares para valorar adherencia, competencia y destrezas; usar medidas de resultado adecuadas, contando con datos de comparación normativos e índices de cambio (estadístico y clínico) significativo y utilizar redes entre investigadores centrados en la práctica clínica.

La evidencia basada en la práctica se puede aplicar a diferentes niveles: desde una consulta individual de un psicólogo clínico que monitoriza y revisa sus resultados, hasta la creación de Practice Research Networks, grupos de clínicos individuales, equipos y servicios que se ponen de acuerdo para compartir una cantidad grande de datos de su práctica rutinaria (Barkham y Mellor-Clark, 2003). Existen investigaciones que muestran que aplicar principios como los que se han venido delineando logra, a largo plazo, mejorar los resultados globales de servicios de salud mental (Reese et al., 2014; Goldberg et al., 2016).

Ejemplos de estudios que podrían categorizarse como evidencia basada en la práctica se encuentran en el trabajo de Bergström et al. (2018) sobre el Diálogo Abierto, en el que describen en detalle la intervención realizada y hacen un se- 
guimiento de los resultados a lo largo de un amplio período de tiempo. También podría ser el caso, más cercano, de la labor de los psicólogos clínicos que trabajan en Atención Primaria en los servicios públicos del Principado de Asturias que, sin ceñirse a un manual o protocolo fijo, han monitorizado sus resultados (recogiendo también feedback cualitativo de los consultantes) y registrado el tipo de intervenciones realizadas (López et al., 2019), complementario a otro tipo de tratamientos más estructurados (véase, por ejemplo, Gárriz et al., 2019) y centrados en variables y procesos psicológicos transdiagnósticos que pretenden resolver algunos de los problemas asociados al modelo médico en psicoterapia.

\section{Conclusiones}

La PBEP ha permitido dotar a la psicoterapia de la entidad que merece, disipando las dudas que acerca de su utilidad se han venido plateando desde algunos sectores. Sin embargo, sigue habiendo aspectos mejorables. El primero es el de la manida comparación con el modelo médico a la hora de adaptar sus propias reglas del juego en cuanto a métodos para mostrar su eficacia. Quizás en las décadas venideras, se observe el desarrollo de una PBEP donde se tenga más en cuenta lo propiamente psicológico y que no necesite calcar las directrices de la medicina basada en la evidencia para gozar de buena autoestima y salud. Otro aspecto importante tiene que ver con el riesgo de centrar toda la atención en los modelos de tratamiento y no en otras variables cuya influencia en una buena evolución de la terapia psicológica es igual o más importante. Circunscribir la práctica clínica al uso de manuales de tratamiento destinados a abordar trastornos conlleva el riesgo de desatender otros aspectos clave, principalmente aquellos relativos a las diferencias individuales (tanto de consultantes como de clínicos), amén de seguir ofreciendo una visión de los problemas psicológicos como equivalentes a los médicos y propiciar el uso interesado de las marcas registradas (Gimeno-Peón et al., 2018a).

El desarrollo de la evidencia basada en la práctica no tiene como finalidad sustituir el paradigma de la PBEP, sino tender puentes entre ambas, y es acorde con lo que para muchos es la clave para ofrecer el mejor servició a la población: más que ofrecer el mismo tratamiento para todo el mundo, una terapia eficaz es aquella que se realiza a la medida de cada persona; es única en cada caso y se guía por las características, fortalezas, preferencias y personalidad de los individuos. Por su parte, el psicólogo clínico efectivo será aquel con una formación que realmente le permita impulsar su desarrollo profesional, humilde y capaz de adaptar tanto el método de tratamiento como el tipo de alianza terapéutica construida con cada persona y circunstancia particular (Norcross y Lambert, 2019). 


\section{Referencias}

American Psychological Association (2006). Evidence-based practice in psychology: APA presidential task force on evidence-based practice. American Psychologist, 61(4), 271-285.

American Psychological Association (2016). Psychological Treatments: Division 12. https://www.div12.org/ treatments/

Anderson, T., Ogles, B. M., Patterson, C. L., Lambert, M. J. y Vermeersch, D. A. (2009). Therapist effects: Facilitative interpersonal skills as a predictor of therapist success. Journal of Clinical Psychology, 65(7), 755-768. https://doi.org/10.1002/jclp.20583

Barkham, M., Lutz, W., Lambert, M. J. y Saxon, D. (2017). Therapist effects, effective therapists, and the law of variability. En L. G. Castonguay y C. E. Hill (eds.), How and why are some therapists better than others?: Understanding therapist effects (pp. 13-36). American Psychological Association. https://doi. org/10.1037/0000034-002

Barkham, M. y Mellor-Clark, J. (2003). Bridging evidence-based practice and practice-based evidence: Developing a rigorous and relevant knowledge for the psychological therapies. Clinical Psychology \& Psychotherapy: An International Journal of Theory \& Practice, 10(6), 319-327. https://doi.org/10.1002/cpp.379

Bennett-Levy, J. (2019). Why therapists should walk the talk: The theoretical and empirical case for personal practice in therapist training and professional development. Journal of behavior therapy and experimental psychiatry, 62, 133-145. https://doi.org/10.1016/j.jbtep.2018.08.004

Berg, H. (2019). Evidence-Based Practice in Psychology Fails to Be Tripartite: A Conceptual Critique of the Scientocentrism in Evidence-Based Practice in Psychology. Frontiers in psychology, 10, 2253-2253. https:// doi.org/10.3389/fpsyg.2019.02253

Berg, H. y Slaattelid, R. (2017). Facts and values in psychotherapy-A critique of the empirical reduction of psychotherapy within evidence-based practice. Journal of Evaluation in Clinical Practice, 23(5), 1075 1080. https://doi.org/10.1111/jep.12739

Bergström, T., Seikkula, J., Alakare, B., Mäki, P., Köngäs-Saviaro, P., Taskila, J. J., Tolvanen, A. y Aaltonen, J. (2018). The family-oriented open dialogue approach in the treatment of first-episode psychosis: Nineteen-year outcomes. Psychiatry research, 270, 168-175. https://doi.org/10.1016/j.psychres.2018.09.039

Beutler, L. E., Edwards, C. y Someah, K. (2018a). Adapting psychotherapy to patient reactance level: A metaanalytic review. Journal of Clinical Psychology, 74(11), 1952-1963. https://doi.org/10.1002/jclp.22682

Beutler, L. E., Kimpara, S., Edwards, C. J. y Miller, K. D. (2018b). Fitting psychotherapy to patient coping style: A meta-analysis. Journal of Clinical Psychology, 74(11), 1980-1995. https://doi.org/10.1002/jclp.22684

Bohart, A. C. y Wade, A. G. (2013). The client in psychotherapy. En M. J. Lambert (ed.), Bergin and Garfield's handbook of psychotherapy and behavior change (pp. 219-257). Wiley.

Castonguay, L., Constantino, M. J. y Xiao, H. (2019). Integrating research and practice. En J. C. Norcross y M. R. Goldfried (eds.), Handbook of psychotherapy integration (pp. 432-447). Oxford University Press.

Castonguay, L. y Hill, C. E. (2017). How and why are some therapists better than others? Understanding therapist effects. American Psychological Association.

Cecchin, G. (2010). Los prejuicios sistémicos [Entrada en un Blog]. http://terapia-familiar-ecosistemica.blogspot. com/2010/03/los-prejuicios-sistemicos.html

Chow, D. (2014). The study of supershrinks: Development anddeliberatepractices of highlyeffective psychotherapists [Tesis Doctoral, Universidad de Curtin (Australia)]. https://espace.curtin.edu.au/handle/20.500.11937/45

Cuijpers, P. (2019). Targets and outcomes of psychotherapies for mental disorders: an overview. World Psychiatry, 18(3), 276-285. https://doi.org/10.1002/wps.20661.

Cuijpers, P., Reijnders, M. y Huibers, M. J. (2019). The role of common factors in psychotherapy outcomes. Annual Review of Clinical Psychology, 15, 207-231. https://doi.org/10.1146/annurev-clinpsy-050718-095424

De Smet, M. M., Meganck, R., De Geest, R., Norman, U. A., Truijens, F. y Desmet, M. (2020). What "good outcome” means to patients: Understanding recovery and improvement in psychotherapy for major depression from a mixed-methods perspective. Journal of Counselling Psychology, 67(1), 25-39. https:// doi.org/10.1037/cou0000362

Duncan, B. L. (2014). On becoming a better therapist. American Psychological Association.

Erickson, K. A., Krampe, R. T. y Tesch-Römer, C. (1993). The role of deliberate practice in the acquisition of expert performance. Psychological review, 100(3), 363-406.

Fernández-Álvarez, J., Prado-Abril, J., Sánchez-Reales, S., Molinari, G., Penedo, J. M. G. y Youn, S. J. L. (2020). La brecha entre la investigación y la práctica clínica. Papeles del Psicólogo, 41(2), 81-90. https://doi. org/10.23923/pap.psicol2020.2932 
Gárriz, M., Elices, M., Peretó, M., Martín-López, L. M., Justicia, A. y Pérez, V. (2020). Mindfulness-based cognitive therapy delivered in primary care: a naturalistic, mixed-methods study of participant characteristics and experiences. Mindfulness, 11(2), 291-302. https://doi.org/10.1007/s12671-019-01166-y

Gimeno-Peón, A., Barrio-Nespereira, A. y Álvarez-Casariego, M. T. (2018a). Psicoterapia: marca registrada. Revista de la Asociación Española de Neuropsiquiatría, 38(133), 131-144.

Gimeno-Peón, A., Barrio-Nespereira, A. y Prado-Abril, J. (2018b). Monitorización sistemática y feedback en psicoterapia. Papeles del Psicólogo, 39(3), 174-182. https://doi.org/10.23923/pap.psicol2018.2872

Goldberg, S. B., Babins-Wagner, R., Rousmaniere, T., Berzins, S., Hoyt, W. T., ... Wampold, B. E. (2016). Creating a climate for therapist improvement: A case study of an agency focused on outcomes and deliberate practice. Psychotherapy, 53(3), 367-375. https://doi.org/10.1037/pst0000060

Goldfried, M. R. (2019). Obtaining consensus in psychotherapy: What holds us back? American Psychologist, 74(4), 484-496. https://doi.org/10.1037/amp0000365

González-Blanch, C. y Carral-Fernández, L. (2017). ¡Enjaulad a Dodo, por favor! El cuento de que todas las psicoterapias son igual de eficaces. Papeles del Psicólogo, 38(2), 94-106. https://doi.org/10.23923/pap. psicol2017.2828

Heinonen, E. y Nissen-Lie, H. A. (2020). The professional and personal characteristics of effective psychotherapists: A systematic review. Psychotherapy Research, 30(4), 417-432. https://doi.org/10.1080/1050330 7.2019.1620366

Krebs, P., Norcross, J. C., Nicholson, J. M. y Prochaska, J. O. (2018). Stages of change and psychotherapy outcomes: A review and meta-analysis. Journal of Clinical Psychology, 74(11), 1964-1979. https://doi. org/10.1002/jclp.22683

Lambert, M. J. (2013). The efficacy and effectiveness of psychotherapy. En M. J. Lambert (ed.), Bergin and Garfield's handbook of psychotherapy and behavior change (pp. 169-218). John Wiley \& Sons.

Langkaas, T. F., Wampold, B. E. y Hoffart, A. (2018). Five types of clinical difference to monitor in practice. Psychotherapy, 55(3), 241-254. https://doi:10.1037/pst0000194

Levy, K. N., Kivity, Y., Johnson, B. N. y Gooch, C. V. (2018). Adult attachment as a predictor and moderator of psychotherapy outcome: A meta-analysis. Journal of Clinical Psychology, 74(11), 1996-2013. https://doi. org/10.1002/jclp.22685

López, M. G., Alonso, N. L., Gómez, R. A. y Martínez, C. V. (2019). Psicología Clínica en Atención Primaria: la experiencia en Asturias. Semergen: revista española de medicina de familia, 2, 101-106. https://doi. org/10.1016/j.semerg.2019.09.002

Luborsky, L., Rosenthal, R., Diguer, L., Andrusyna, T. P., Berman, J. S., Levitt, J. T., Seligman, D. A. y Krause, E. D. (2002). The dodo bird verdict is alive and well-mostly. Clinical Psychology: Science and Practice, 9(1), 2-12. https://doi.org/10.1093/clipsy.9.1.2

Margison, F. R., Barkham, M., Evans, C., McGrath, G., Clark, J. M., Audin, K. y Connell, J. (2000). Measurement and psychotherapy: Evidence-based practice and practice-based evidence. The British Journal of Psychiatry, 177(2), 123-130. https://doi.org/10.1192/bjp.177.2.123

Mulder, R., Murray, G. y Rucklidge, J. (2017). Common versus specific factors in psychotherapy opening the black box. The Lancet Psychiatry, 4(12), 953-962. https://doi.org/10.1016/S2215-0366(17)30100-1

Montejano, S. R. y García, A. M. (2019). Reflexiones sobre la formación en Psicología Clínica: el camino hacia la Pericia. Clínica Contemporánea, 3(10), 1-15. https://doi.org/10.5093/cc2019a19

Nissen-Lie, H. A., Rønnestad, M. H., Høglend, P. A., Havik, O. E., Solbakken, O. A., Stiles, T. C. y Monsen, J. T. (2017). Love yourself as a person, doubt yourself as a therapist? Clinical Psychology \& Psychotherapy, 24(1), 48-60. https://doi.org/10.1002/cpp.1977

Norcross, J. C. y Lambert, M. J. (2019). Evidence-based psychotherapy relationships: The third task force. En J. C. Norcross y M. J. Lambert (eds.), Psychotherapy relationships that work: Volume 1: Evidence-based therapist contributions (pp. 1-23). Oxford University Press.

Norcross, J. C. y Wampold, B. E. (2018). A new therapy for each patient: Evidence-based relationships and responsiveness. Journal of Clinical Psychology, 74(11), 1889-1906. https://doi.org/10.1002/jclp.22678

Paris, J. (2013). How the history of psychotherapy interferes with integration. Journal of Psychotherapy Integration, 23(2), 99-106. https://doi.org/10.1037/a0031419

Pérez-Álvarez, M. (2019). La psicoterapia como ciencia humana, más que tecnológica. Papeles del Psicólogo, 40(1), 1-14. https://doi.org/10.23923/pap.psicol2019.2877

Pérez-Álvarez, M., Fernández Hermida, J. R., Fernández Rodríguez, C. y Amigo Vázquez I. (2003). Guía de tratamientos psicológicos eficaces I. Ediciones Pirámide. 
Prado-Abril, J., Fernández-Álvarez, J., Sánchez-Reales, S., Youn, S. J., Inchausti, F. y Molinari, G. (2019). La persona del terapeuta: Validación española del Cuestionario de Evaluación del Estilo Personal del Terapeuta (EPT-C). Revista de Psicopatología y Psicología Clínica, 24(3), 131-140. https://doi.org/10.5944/rppc.24367

Prado-Abril, J., Gimeno-Peón, A., Inchausti, F. y Sánchez-Reales, S. (2019). Pericia, efectos del terapeuta y práctica deliberada: El ciclo de la excelencia. Papeles del Psicólogo, 40(2), 89-100. https://doi.org/10.23923/ pap.psicol2019.2888

Prado-Abril, J., Sánchez-Reales, S. y Inchausti, F. (2017). En busca de nuestra mejor versión: pericia y excelencia en Psicología Clínica. Ansiedad y Estrés, 23(2-3), 110-117. https://doi.org/10.1016/j.anyes.2017.06.001

Reese, R. J., Duncan, B. L., Bohanske, R. T., Owen, J. J. y Minami, T. (2014). Benchmarking outcomes in a public behavioral health setting: Feedback as a quality improvement strategy. Journal of Consulting and Clinical Psychology, 82(4), 731-742. https://doi.org/10.1037/a0036915

Roe, D., Dekel, R., Harel, G. y Fennig, S. (2006). Clients' reasons for terminating psychotherapy: A quantitative and qualitative inquiry. Psychology and Psychotherapy: Theory, Research and Practice, 79(4), 529-538. https://doi.org/10.1348/147608305X90412

Rousmaniere, T. (2016). Deliberate practice for psychotherapists: A guide to improving clinical effectiveness. Taylor \& Francis. https://doi.org/10.4324/9781315472256

Rousmaniere, T. (2019). Mastering the Inner skills of psychotherapy: A deliberate practice manual. Gold Lantern Books.

Sakaluk, J. K., Williams, A. J., Kilshaw, R. E. y Rhyner, K. T. (2019). Evaluating the evidential value of empirically supported psychological treatments (ESTs): A meta-scientific review. Journal of Abnormal Psychology, 128(6), 500-509. https://doi.org/10.1037/abn0000421

Slife, B. D., Burchfield, C. y Hedges, D. (2010). Interpreting the "biologization" of psychology. The Journal of Mind and Behavior, 31(3) 165-177.

Soto, A., Smith, T. B., Griner, D., Domenech Rodríguez, M. y Bernal, G. (2018). Cultural adaptations and therapist multicultural competence: Two meta-analytic reviews. Journal of Clinical Psychology, 74(11), 1907-1923. https://doi.org/10.1002/jclp.22679

Stedmon, J. y Dallos, R. (2009). Reflective practice in psychotherapy and counselling. McGraw-Hill Education.

Swift, J. K., Callahan, J. L., Cooper, M. y Parkin, S. R. (2018). The impact of accommodating client preference in psychotherapy: A meta-analysis. Journal of Clinical Psychology, 74(11), 1924-1937. https://doi. org/10.1002/jclp.22680

Swift, J. K. y Greenberg, R. P. (2012). Premature discontinuation in adult psychotherapy: A meta-analysis. Journal of Consulting and Clinical Psychology, 80(4), 547-559. https://doi.org/10.1037/a0028226

Tackett, J. L., Brandes, C. M., King, K. M. y Markon, K. E. (2019). Psychology’s replication crisis and clinical psychological science. Annual Review of Clinical Psychology, 15, 579-604. https://doi.org/10.1146/ annurev-clinpsy-050718-095710

Wampold, B. E., Baldwin, S. A., Holtforth, M. G. e Imel, Z. E. (2017). What characterizes effective therapists? En L. G. Castonguay y C. E. Hill (eds.), How and why are some the-rapists better than others? (pp. 37-54). American Psychological Association. https://doi.org/10.1037/0000034-003

Wampold, B. E. e Imel, Z. E. (2015). The great psychotherapy debate: The evidence for what makes psychotherapy work. Routledge.

Westmacott, R. y Hunsley, J. (2010). Reasons for terminating psychotherapy: A general population study. Journal of Clinical Psychology, 66(9), 965-977. https://doi.org/10.1002/jclp.20702 